\title{
A BÄCKLUND TRANSFORMATION AND NONLINEAR SUPERPOSITION FORMULA FOR THE LOTKA-VOLTERRA HIERARCHY
}

\author{
XING-BIAO HU ${ }^{12}$ and JOHAN SPRINGAEL ${ }^{3}$
}

(Received 24 May, 2000)

\begin{abstract}
A hierarchy of bilinear Lotka-Volterra equations with a unified structure is proposed. The bilinear Bäcklund transformation for this hierarchy and the corresponding canonical Lax pair are obtained. Furthermore, the nonlinear superposition formula is proved rigorously.
\end{abstract}

\section{Introduction}

Recursion operators and Hirota bilinear forms have played an important role in the development of soliton theory. Recursion operators were first introduced by Olver in 1977 [23] and developed by Fuchssteiner [5] and by Fokas and Santini [4, 24], while Hirota bilinear forms were introduced by Hirota in 1971 [6]. By using recursion operators, we can easily generate a hierarchy of integrable equations. However, recursion operators cannot be applied directly to bilinear equations. Instead recursion operators are characterized by bilinear equations with a unified structure (or canonical form). For example, starting from the isospectral problem

$$
\Psi_{x}=\left(\begin{array}{cc}
-i \lambda & q(x, t) \\
r(x, t) & i \lambda
\end{array}\right) \Psi, \quad \Psi=\left(\begin{array}{l}
\psi_{1} \\
\psi_{2}
\end{array}\right)
$$

\footnotetext{
'Academy of Mathematics and Systems Sciences, State Key Laboratory of Scientific and Engineering Computing, Institute of Computational Mathematics and Scientific Engineering Computing, Academia Sinica, P.O. Box 2719, Beijing 100080, P.R. China; e-mail: hxb@1sec.cc.ac.cn.

${ }^{2}$ Institute of Mathematics and Statistics, University of Kent at Canterbury, Canterbury CT2 7NF, United Kingdom.

${ }^{3}$ Dienst Theoretische Natuurkunde, Vrije Universiteit Brussel, Pleinlaan 2, B-1050 Brussels, Belgium; e-mail: jspringa@mach.vub.ac.be.

(C) Australian Mathematical Society 2002, Serial-fee code 1446-1811/02
} 
we can obtain the AKNS hierarchy $[1,2,22]$

$$
\left(\begin{array}{l}
q_{t} \\
r_{t}
\end{array}\right)=R^{n}\left(\begin{array}{l}
q_{x} \\
r_{x}
\end{array}\right), \quad n \geq 1
$$

where $R$ is a recursion operator which is given by

$$
R=\frac{1}{2 i}\left(\begin{array}{cc}
-\partial_{x}+2 q \partial_{x}^{-1} r & 2 q \partial_{x}^{-1} q \\
-2 r \partial_{x}^{-1} r & \partial_{x}-2 r \partial_{x}^{-1} q
\end{array}\right)
$$

By introducing an infinite number of variables $x=t_{1}, t_{2}, t_{3}, \ldots$ and considering $q$ and $r$ to be functions of $t=\left(t_{1}, t_{2}, t_{3}, \ldots\right)$ we have the equivalent equation

$$
\left(\begin{array}{l}
q_{t_{n+1}} \\
r_{t_{n+1}}
\end{array}\right)=\frac{1}{2 i}\left(\begin{array}{cc}
-\partial_{x}+2 q \partial_{x}^{-1} r & 2 q \partial_{x}^{-1} q \\
-2 r \partial_{x}^{-1} r & \partial_{x}-2 r \partial_{x}^{-1} q
\end{array}\right)\left(\begin{array}{l}
q_{t_{n}} \\
r_{t_{n}}
\end{array}\right), \quad n \geq 1 .
$$

Using the dependent variable transformation $q=\sigma / \tau, r=\rho / \tau$ one can deduce the bilinear equations, which have a unified structure $[12,22]$ :

$$
\left(D_{t_{n+1}}-\frac{i}{2} D_{t_{1}} D_{t_{n}}\right) \sigma \cdot \tau=0, \quad\left(D_{t_{n+1}}+\frac{i}{2} D_{t_{1}} D_{t_{n}}\right) \rho \cdot \tau=0, \quad D_{t_{1}}^{2} \tau \cdot \tau=-2 \sigma \rho,
$$

where the Hirota bilinear operators $D_{x}^{m} D_{t}^{n}$ are defined as $[8,10,19]$

$$
\left.D_{x}^{m} D_{t}^{n} a(x, t) \cdot b(x, t) \equiv\left(\partial_{x}-\partial_{x^{\prime}}\right)^{m}\left(\partial_{t}-\partial_{t^{\prime}}\right)^{n} a(x, t) b\left(x^{\prime}, t^{\prime}\right)\right|_{x^{\prime}=x, t^{\prime}=t} .
$$

It should be noted that the unified bilinear form (UBF) for the AKNS hierarchy was obtained by Newell [22] without explicit use of the recursion operator.

There are two systematic ways to obtain such a UBF; one way is the so called recursion operator approach $[11,12]$ and the other is based on the structure of the soliton solutions [25]. As a result UBFs for several hierarchies of integrable equations can be obtained. Since UBFs are candidates for recursion operators in bilinear form, it is natural to derive such unified bilinear forms by using recursion operators where they are available. Compared with the second method, this also avoids tedious calculations in testing multi-soliton solutions.

On the other hand, the remarkable advantage in finding UBFs based on the structure of the soliton solutions is that this approach does not depend on knowledge of the recursion operator. This can lead in some cases to an unknown recursion operator.

In this paper, we will generalize UBFs to the case of differential-difference equations. By using a corresponding recursion operator, a UBF for the Lotka-Volterra hierarchy is proposed. To our knowledge, this is the first example in literature giving a UBF in the differential-difference case. Furthermore, a bilinear Bäcklund transformation (BBT) for the Lotka-Volterra hierarchy is presented. From this BBT we obtain the Lax pair for the Lotka-Volterra hierarchy in a concise form. Finally, a nonlinear superposition formula is proved rigorously. 


\section{A UBF for the Lotka-Volterra hierarchy}

The Lotka-Volterra (LV) or Kac-van Moerbeke equation is given by

$$
u_{n, t_{1}}=u_{n}\left(u_{n-1}-u_{n+1}\right)
$$

with $u_{n, f_{1}} \equiv \partial_{t_{1}} u_{n}$. Much work has been performed on (1) and its generalization (see for example $[3,7,9,13-18,20,21,26,27])$. In [27] a recursion operator for (1) was presented in the following form:

$$
R=u_{n}\left(1+T_{-}\right)\left(u_{n} T_{-}-T_{+} u_{n}\right)\left(1-T_{-}\right)^{-1} u_{n}^{-1}
$$

where $T_{ \pm} u_{n}=u_{n \pm 1}$. As a result, higher order LV equations can be written as

$$
u_{n, t_{k}}=R^{k-1} u_{n, t_{1}}, \quad k>1
$$

or equivalently

$$
u_{n, t_{k}}=R u_{n, t_{k-1}}
$$

This equation can be bilinearized using the dependent variable transformation

$$
u_{n}=\frac{f_{n-3 / 2} f_{n+3 / 2}}{f_{n-1 / 2} f_{n+1 / 2}} .
$$

Using this last transformation we have that

$$
\begin{aligned}
\left(1+T_{-}\right)^{-1}\left(u_{n}^{-1} u_{n, t_{k}}\right) & =\frac{f_{n+3 / 2, t_{k}}}{f_{n+3 / 2}}-2 \frac{f_{n+1 / 2, t_{k}}}{f_{n+1 / 2}}+\frac{f_{n-1 / 2, t_{k}}}{f_{n-1 / 2}}, \\
\left(1-T_{-}\right)^{-1}\left(u_{n}^{-1} u_{n, t_{k-1}}\right) & =\frac{f_{n+3 / 2, t_{k-1}}}{f_{n+3 / 2}}-\frac{f_{n-1 / 2, t_{k-1}}}{f_{n-1 / 2}} .
\end{aligned}
$$

Furthermore, (1) and (2) can respectively be transformed into the bilinear equations

$$
\left[D_{t_{1}} \sinh \left(\frac{1}{2} D_{n}\right)+\cosh \left(\frac{3}{2} D_{n}\right)-\cosh \left(\frac{1}{2} D_{n}\right)\right] f_{n} \cdot f_{n}=0
$$

and

$$
\begin{aligned}
D_{t_{k}} f_{n+1 / 2} \cdot f_{n-1 / 2}= & f_{n+3 / 2} f_{n-3 / 2, t_{k-1}}-f_{n+1 / 2, t_{k-1}} f_{n-1 / 2} \\
& -f_{n-1 / 2, t_{1}} f_{n+1 / 2, t_{k-1}}+f_{n+1 / 2, r_{1}, t_{k-1}} f_{n-1 / 2} .
\end{aligned}
$$

Using (3), we can rewrite (4) as

$$
\begin{aligned}
& {\left[D_{t_{k}} \sinh \left(\frac{1}{2} D_{n}\right)+\frac{1}{2} D_{t_{k-1}} \sinh \left(\frac{3}{2} D_{n}\right)\right.} \\
& \left.\quad+\frac{1}{2} D_{t_{k-1}} \sinh \left(\frac{1}{2} D_{n}\right)-\frac{1}{2} D_{t_{1}} D_{t_{k-1}} \cosh \left(\frac{1}{2} D_{n}\right)\right] f_{n} \cdot f_{n}=0,
\end{aligned}
$$

which is nothing but the UBF of the Lotka-Volterra hierarchy. Equations (3) and (5) together constitute the whole hierarchy of LV equations in bilinear form. 


\section{A unified BBT and a unified Lax pair for the Lotka-Volterra hierarchy}

By application of the exchange formalism one can construct, using the necessary exchange formulas (A1)-(A6) (see Appendix for details) a bilinear Bäcklund transformation for (3) and (5),

$$
\begin{gathered}
e^{D_{n}} f_{n} \cdot g_{n}=\lambda f_{n} g_{n}+\mu e^{-D_{n}} f_{n} \cdot g_{n}, \\
\left(D_{t_{1}}-\lambda e^{-D_{n}}-\gamma\right) f_{n} \cdot g_{n}=0, \\
{\left[D_{t_{k}}+\frac{\lambda}{2} D_{t_{k-1}} e^{-D_{n}}-\frac{\lambda}{2 \mu} D_{t_{t-1}} e^{D_{n}}-\frac{\lambda^{2}}{2 \mu} D_{t_{k-1}}\right] f_{n} \cdot g_{n}=0,}
\end{gathered}
$$

where $\lambda, \mu$ and $\gamma$ are arbitrary parameters.

Using the linearizing transformation $\psi_{n}=f_{n} / g_{n}, v_{n}=g_{n+1} g_{n-1} / g_{n}^{2}$ one can transform the unified BBT (6)-(8) into the following Lax pair:

$$
\begin{gathered}
L_{n} \psi_{n} \equiv v_{n} \psi_{n+1}-\lambda \psi_{n}-\mu v_{n} \psi_{n-1}=0, \quad B_{n}^{(1)} \psi_{n} \equiv \psi_{n, t_{1}}-\lambda v_{n} \psi_{n-1}-\gamma \psi_{n}=0 \\
B_{n}^{(k)} \psi_{n} \equiv \psi_{n, t_{k}}-\frac{\lambda^{2}}{\mu} U_{n, t_{k-1}} \psi_{n}-\lambda v_{n}\left(U_{n, t_{k-1}}+U_{n+1, t_{t-1}}\right) \psi_{n-1}-\frac{\lambda^{2}}{\mu} \psi_{n, t_{k-1}}=0
\end{gathered}
$$

with $k>1$ and $U_{n}=\sum_{l=0}^{\infty} \ln \left(v_{n-1-l}\right)$. The compatibility condition

$$
\left(L_{n} B_{n}^{(k)}-B_{n}^{(k)} L_{n}\right) \psi_{n}=0
$$

is satisfied when $u_{n}=v_{n+1 / 2} v_{n-1 / 2}$ satisfies (2).

\section{A nonlinear superposition formula for the Lotka-Volterra hierarchy}

In this section we present a superposition formula for the solutions of the UBF (3) and (5).

PROPOSITION 1. Let $f_{0}$ be a solution of the system given by (3) and (5). Suppose that $f_{i}(i=1,2)$ are two other solutions of (3) and (5) which are related to $f_{0}$ under the unified BBT (6)-(8) with parameters $\left(\lambda_{i}, \mu_{i}, \gamma_{i}\right)$, that is, $f_{0} \stackrel{\left(\lambda_{i}, \mu_{i}, \gamma_{i}\right)}{\longrightarrow} f_{i}(i=1,2)$, where $\lambda_{1} \lambda_{2} \neq 0, f_{j} \neq 0(j=0,1,2)$. Then $f_{12}$ defined by

$$
e^{-\frac{1}{2} D_{n}} f_{0} \cdot f_{12}=c\left[\lambda_{1} e^{-\frac{1}{2} D_{n}}-\lambda_{2} e^{\frac{1}{2} D_{n}}\right] f_{1} \cdot f_{2},
$$

where $c$ is a nonzero constant, is a new solution which is related to $f_{1}$ and $f_{2}$ under the unified BBT (6)-(8) with parameters $\left(\lambda_{2}, \mu_{2}, \gamma_{2}\right)$ and $\left(\lambda_{1}, \mu_{1}, \gamma_{1}\right)$ respectively. 
Proof. Similar to the deduction in [14], we can show that

$$
\begin{gathered}
f_{0} f_{12}=c\left[\mu_{1} e^{D_{n}}+\mu_{2} e^{-D_{n}}\right] f_{1} \bullet f_{2}, \\
-D_{t_{1}} f_{1} \cdot f_{2}+\left(\gamma_{2}-\gamma_{1}\right) f_{1} f_{2}-(1 / c) e^{-D_{n}} f_{0} \bullet f_{12}=0, \\
{\left[e^{D_{n}}-\lambda_{j}-\mu_{j} e^{-D_{n}}\right] f_{i} \cdot f_{12}=0, \quad i \neq j, \quad i, j=1,2,} \\
{\left[D_{t_{1}}-\lambda_{j} e^{-D_{n}}-\gamma_{j}\right] f_{i} \cdot f_{12}=0, \quad i \neq j, \quad i, j=1,2 .}
\end{gathered}
$$

In order to prove Proposition 1, it suffices to show that

$$
\left[D_{t_{k}}+\frac{\lambda_{i}}{2} D_{t_{t-1}} e^{-D_{n}}-\frac{\lambda_{i}}{2 \mu_{i}} D_{t_{k-1}} e^{D_{n}}-\frac{\lambda_{i}^{2}}{2 \mu_{i}} D_{t_{k-1}}\right] f_{j} \cdot f_{12}=0, \quad i \neq j, i, j=1,2 .
$$

Since $f_{1}$ and $f_{2}$ are two solutions of (3) and (5), we have that

$$
\begin{aligned}
{\left[e^{\frac{1}{2} D_{n}}\right.} & \left.f_{2} \cdot f_{2}\right]\left\{\left[D_{t_{k}} \sinh \left(\frac{1}{2} D_{n}\right)+\frac{1}{2} D_{t_{k-1}} \sinh \left(\frac{3}{2} D_{n}\right)+\frac{1}{2} D_{t_{k-1}} \sinh \left(\frac{1}{2} D_{n}\right)\right.\right. \\
& \left.\left.-\frac{1}{2} D_{t_{1}} D_{t_{k-1}} \cosh \left(\frac{1}{2} D_{n}\right)\right] f_{1} \cdot f_{1}\right\}-\left\{\left[D_{t_{k}} \sinh \left(\frac{1}{2} D_{n}\right)+\frac{1}{2} D_{t_{k-1}} \sinh \left(\frac{3}{2} D_{n}\right)\right.\right. \\
& \left.\left.+\frac{1}{2} D_{t_{k-1}} \sinh \left(\frac{1}{2} D_{n}\right)-\frac{1}{2} D_{t_{1}} D_{t_{k-1}} \cosh \left(\frac{1}{2} D_{n}\right)\right] f_{2} \cdot f_{2}\right\}\left[e^{\frac{1}{2} D_{n}} f_{1} \cdot f_{1}\right] \\
& -\frac{1}{2}\left[D_{t_{k-1}} e^{\frac{1}{2} D_{n}} f_{2} \bullet f_{2}\right]\left[D_{t_{1}} \sinh \left(\frac{1}{2} D_{n}\right)+e^{\frac{3}{2} D_{t}}-e^{\frac{1}{2} D_{n}}\right] f_{1} \bullet f_{1} \\
& +\frac{1}{2}\left[D_{t_{k-1}} e^{\frac{1}{2} D_{n}} f_{1} \bullet f_{1}\right]\left[D_{t_{1}} \sinh \left(\frac{1}{2} D_{n}\right)+e^{\frac{3}{2} D_{n}}-e^{\frac{1}{2} D_{n}}\right] f_{2} \bullet f_{2}=0 .
\end{aligned}
$$

Using formulas (A1)-(A10), (9)-(11) we can rewrite (13) as

$$
-\frac{1}{c \lambda_{i}} e^{-\frac{1}{2} D_{n}^{\prime}} f_{0} f_{i} \cdot\left\{\left[D_{t_{k}}+\frac{\lambda_{i}}{2} D_{t_{k-1}} e^{-D_{n}}-\frac{\lambda_{i}}{2 \mu_{i}} D_{t_{k-1}} e^{D_{n}}-\frac{\lambda_{i}^{2}}{2 \mu_{i}} D_{t_{k-1}}\right] f_{j} \cdot f_{12}\right\}=0,
$$

for $i \neq j, i, j=1,2$, which means that (12) holds. Therefore we complete the proof of Proposition 1.

As an application of the nonlinear superposition formula (9), we shall now construct soliton solutions of the Lotka-Volterra hierarchy in bilinear form. Choose for example $f_{0}=1, c=1 /\left(\lambda_{1}-\lambda_{2}\right)$. It is easily verified that

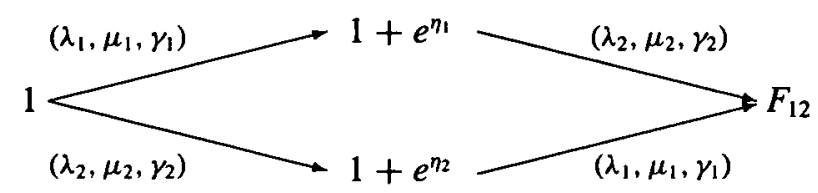

where

$$
F_{12}=1+\frac{\lambda_{1}-\lambda_{2} e^{2 p_{1}}}{\lambda_{1}-\lambda_{2}} e^{2 \eta_{1}}+\frac{\lambda_{2}-\lambda_{1} e^{2 p_{2}}}{\lambda_{2}-\lambda_{1}} e^{2 \eta_{2}}+\frac{\lambda_{1} e^{2 p_{2}}-\lambda_{2} e^{2 p_{1}}}{\lambda_{1}-\lambda_{2}} e^{2\left(\eta_{1}+\eta_{2}\right)}
$$

with $\eta_{i}=-p_{i} n+\omega_{i}^{(1)} t_{1}+\cdots+\omega_{i}^{(k)} t_{k}+\cdots, \omega_{i}^{(1)}=\sinh \left(2 p_{i}\right), \lambda_{i}=1+e^{2 p_{i}}, \mu_{i}=-e^{2 p_{i}}$, $\gamma_{i}=-\left(1+e^{2 p_{i}}\right)$ and $\omega_{i}^{(k)}=(-1)^{k-1} e^{-2(k-1) p_{i}}\left(1+e^{2 p_{i}}\right)^{2(k-1)} \sinh \left(2 p_{i}\right)$. In general, along these lines, we can obtain multisoliton solutions for the Lotka-Volterra hierarchy (3) and (5) step by step. 


\section{Conclusion}

In this paper we derived a UBF for the Lotka-Volterra hierarchy through the bilinearization of the recursion operator. Applying the exchange formalism we obtained the corresponding unified BBT leading to a unified Lax pair. Finally, we proved a nonlinear superposition formula for this Lotka-Volterra hierarchy.

\section{Acknowledgements}

X. B. Hu would like to thank N. Joshi for the invitation and support to attend the Kruskal 2000 meeting. The results of the present paper were obtained when the first author was visiting the Free University of Brussels. He is grateful to F. Lambert and TENA of the Free University of Brussels for the warm hospitality he received during his short visit. He also greatly acknowledges the support by the Royal Society, the Chinese Academy of Sciences and the National Natural Science Foundation of China (Project no. 10171100). J. S. wishes to acknowledge financial support extended within the framework of the "Interuniversity Poles of Attraction Programme, contract no. P4/08Belgian State, Prime Minister's Office-Federal Office for Scientific, Technical and Cultural Affairs".

\section{Appendix A. Hirota bilinear operator identities}

The following bilinear operator identities hold for arbitrary functions $a, b, c$ and $d$ :

$$
\begin{gathered}
{\left[e^{\delta D_{n}} b \cdot b\right]\left[D_{z} \sinh \left(\delta D_{n}\right) a \cdot a\right]-\left[e^{\delta D_{n}} a \cdot a\right]\left[D_{z} \sinh \left(\delta D_{n}\right) b \cdot b\right]} \\
\quad=2 \sinh \left(\delta D_{n}\right)\left(D_{z} a \cdot b\right) \cdot a b, \\
{\left[D_{y} D_{t} e^{\frac{1}{2} D_{n}} a \cdot a\right]\left[e^{\frac{1}{2} D_{n}} b \cdot b\right]-\left[D_{y} D_{t} e^{\frac{1}{2} D_{n}} b \cdot b\right]\left[e^{\frac{1}{2} D_{n}} a \cdot a\right]} \\
-\left[D_{y} e^{\frac{1}{2} D_{n}} a \cdot a\right]\left[D_{t} e^{\frac{1}{2} D_{n}} b \cdot b\right]+\left[D_{y} e^{\frac{1}{2} D_{n}} b \cdot b\right]\left[D_{t} e^{\frac{1}{2} D_{n}} a \cdot a\right] \\
=2 D_{y} \cosh \left(\frac{1}{2} D_{n}\right)\left(D_{t} a \cdot b\right) \cdot a b, \\
{\left[D_{y} e^{\frac{3}{2} D_{n}} a \cdot a\right]\left[e^{\frac{1}{2} D_{n}} b \cdot b\right]-\left[D_{y} e^{\frac{3}{2} D_{n}} b \cdot b\right]\left[e^{\frac{1}{2} D_{n}} a \cdot a\right]} \\
\quad-\left[e^{\frac{3}{2} D_{n}} a \cdot a\right]\left[D_{y} e^{\frac{1}{2} D_{n}} b \cdot b\right]+\left[D_{y} e^{\frac{1}{2} D_{n}} a \cdot a\right]\left[e^{\frac{3}{2} D_{n}} b \cdot b\right] \\
\quad=2 D_{y} \cosh \left(\frac{1}{2} D_{n}\right)\left[e^{D_{n}} a \cdot b\right] \cdot\left[e^{-D_{n}} a \cdot b\right], \\
\quad \sinh \left(\delta D_{n}\right) a \cdot a=0, \\
D_{y} \cosh \left(\frac{1}{2} D_{n}\right)\left[e^{-D_{n}} a \cdot b\right] \cdot a b \\
=-\sinh \left(\frac{1}{2} D_{n}\right)\left\{\left[D_{y} e^{-D_{n}} a \cdot b\right] \cdot a b+\left[D_{y} a \cdot b\right] \cdot\left[e^{-D_{n}} a \cdot b\right]\right\}, \\
D_{y} \cosh \left(\frac{1}{2} D_{n}\right)\left[e^{D_{n}} a \cdot b\right] \cdot a b \\
\quad=\sinh \left(\frac{1}{2} D_{n}\right)\left\{\left[D_{y} e^{D_{n}} a \cdot b\right] \cdot a b+\left[D_{y} a \cdot b\right] \cdot\left[e^{D_{n}} a \cdot b\right]\right\},
\end{gathered}
$$




$$
\begin{aligned}
2 \sinh \left(\delta D_{n}\right)\left(D_{t} a \cdot b\right) \cdot a b=D_{t}\left[e^{\delta D_{n}} a \cdot b\right] \cdot\left[e^{-\delta D_{n}} a \cdot b\right], \\
D_{t}\left[e^{\delta D_{n}} a \cdot b\right] \cdot\left[e^{-\delta D_{n}} c \cdot d\right]=e^{\delta D_{n}}\left[\left(D_{t} a \cdot d\right) \cdot b c-a d \cdot\left(D_{t} c \cdot b\right)\right], \\
2 D_{y} \cosh \left(\frac{1}{2} D_{n}\right)\left[e^{-D_{n}} a \cdot b\right] \cdot c d \\
=e^{-\frac{1}{2} D_{n}}\left\{\left[D_{y} e^{-D_{n}} a \cdot d\right] \cdot c b-a d \cdot\left[D_{y} e^{-D_{n}} c \cdot b\right]\right. \\
\left.+\left[D_{y} a \cdot d\right] \cdot\left[e^{-D_{n}} c \cdot b\right]-\left[e^{-D_{n}} a \cdot d\right] \cdot\left[D_{y} c \cdot b\right]\right\} \\
2 D_{y} \cosh \left(\frac{1}{2} D_{n}\right) a b \cdot\left[e^{D_{n}} c \cdot d\right] \\
=e^{-\frac{1}{2} D_{n}}\left\{\left[D_{y} e^{D_{n}} a \cdot d\right] \cdot c b-a d \cdot\left[D_{y} e^{D_{n}} c \cdot b\right]\right. \\
\left.+\left[D_{y} a \cdot d\right] \cdot\left[e^{D_{n}} c \cdot b\right]-\left[e^{D_{n}} a \cdot d\right] \cdot\left[D_{y} c \cdot b\right]\right\}
\end{aligned}
$$

\section{References}

[1] M. J. Ablowitz and H.Segur, Solitons and the Inverse Scattering Transform (SIAM, Philadelphia, 1981).

[2] M. J. Ablowitz, D. J. Kaup, A. C. Newell and H.Segur, "The inverse scattering transform-Fourier analysis for nonlinear problems", Stud. Appl. Math. 53 (1974) 249-315.

[3] O. I. Bogoyavlensky, "Integrable discretizations of the KdV equation", Phys. Lett. A 134 (1988) 34-38.

[4] A. S. Fokas and P. M. Santini, "Recursion operators and bi-Hamiltonian structures in multidimensions. II", Comm. Math. Phys. 116 (1988) 449-474.

[5] B. Fuchssteiner, "Application of hereditary symmetries to nonlinear evolution equations", Nonlinear Anal. Theory Meth. Appl. 3 (1979) 849-862.

[6] R. Hirota, "Exact solution of the Korteweg-de Vries equation for multiple collisions of solitons", Phys. Rev. Lett. 27 (1971) 1192-1194.

[7] R. Hirota, "Nonlinear partial difference equations I. A difference analogue of the Korteweg-de Vries equation", J. Phys. Soc. Japan 43 (1977) 1424-1433.

[8] R. Hirota, "Direct methods in soliton theory", in Solitons (eds. R. K. Bullough and P. J. Caudrey), (Springer, Berlin, 1980).

[9] R. Hirota and J. Satsuma, " $N$-soliton solutions of nonlinear network equations describing a Volterra system”, J. Phys. Soc. Japan 40 (1976) 891-900.

[10] R. Hirota and J. Satsuma, "A variety of nonlinear network equations generated from the Bäcklund transformation for the Toda lattice", Prog. Theor. Phys. Suppl. 59 (1976) 64-100.

[11] X. B. Hu, "Integrable systems and related problems", Ph. D. Thesis, Computing Center of Academia Sinica, 1990.

[12] X. B. Hu, "Bilinearization of nonlinear integrable evolution equations-Recursion operator approach", preprint, Computing Center of Academia Sinica, 1991.

[13] X. B. Hu, "Nonlinear superposition formulae for the differential-difference analogue of the KdV equation and two dimensional Toda equation", J. Phys. A: Math. Gen. 27 (1994) 201-214.

[14] X. B. Hu and R. K. Bullough, "Bäcklund transformation and nonlinear superposition formula of an extended Lotka-Volterra equation", J. Phys. A: Math. Gen. 30 (1997) 3635-3641.

[15] X. B. Hu and P. A. Clarkson, "Rational solutions of a differential-difference KdV equation, the Toda equation and the discrete KdV equation", J. Phys. A: Math. Gen. 28 (1995) 5009-5016.

[16] Y. Itoh, "On a ruin problem with interaction", Ann. Inst. Stat. Math. 25 (1973) 635-641. 
[17] Y. Itoh, "Integrals of a Lotka-Volterra system of odd number of variables", Prog. Theor. Phys. 78 (1987) 507-510.

[18] S. V. Manakov, "Complete integrability and stochastization of discrete dynamical systems", Sov. Phys. JETP 40 (1974) 269-274.

[19] Y. Matsuno, Bilinear Transformation Method (Academic Press, New York, 1984).

[20] A. Nagai and J. Satsuma, "The Lotka-Volterra equations and the QR algorithm", J. Phys. Soc. Japan 64 (1995) 3669.

[21] K. Narita, "Soliton solution to extended Volterra equations", J. Phys. Soc. Japan 51 (1982) 1682.

[22] A. C. Newell, Solitons in Mathematics and Physics (SIAM, Philadelphia, 1985).

[23] P. J. Olver, "Evolution equations possessing infinitely many symmetries", J. Math. Phys. 18 (1977) 1212-1215.

[24] P. M. Santini and A. S. Fokas, "Recursion operators and bi-Hamiltonian structures in multidimensions. I", Comm. Math. Phys. 115 (1988) 375-419.

[25] R. Willox, F. Lambert and J. Springael, "Canonical bilinear systems and soliton resonances", in Application of Analytic and Geometric Methods to Nonlinear Differential Equations (ed. P. A. Clarkson), (Kluwer, Dordrecht, 1993) 257-270.

[26] V. E. Zakharov, S. L. Musher and A. M. Rubenchik, "Nonlinear stage of parametric wave excitation in plasma", JETP. Lett. 19 (1974) 151-153.

[27] H. W. Zhang, G. Z. Tu, W. Oevel and B. Fuchssteiner, "Symmetries, conserved quantities, and hierarchies for some lattice systems with soliton structure", J. Math. Phys. 32 (1991) 1908-1918. 